\title{
EFEITO AGUDO DO ALONGAMENTO ESTÁTICO E AQUECIMENTO ESPECÍFICO NO DESEMPENHO DO NÚMERO DE REPETIÇÕES E PERCEPÇÃO SUBJETIVA DE ESFORÇO EM MULHERES
}

\section{ACUTE EFFECT OF STATIC STRETCHING AND SPECIFIC WARMING UP ON PERFORMANCE OF THE NUMBER OF REPETITIONS AND PERCEIVED EXERTION IN WOMEN}

\author{
Vitor Correia De Andradel, Júlio César Gomes da Silva", Lucas Dantas Maia Forte ${ }^{I I I}$, Gabriel Rodrigues Neto ${ }^{\text {II** }}$
}

\begin{abstract}
Resumo. A prática do alongamento muscular e do aquecimento, antes ou depois do exercício, tem provocado controvérsias no âmbito científico em relação aos seus benefícios, ou prejuízos, no que diz respeito ao desempenho muscular do participante. Assim, o objetivo do presente estudo foi analisar o efeito agudo do alongamento estático e aquecimento específico no desempenho do número de repetições e percepção subjetiva de esforço (PSE) em mulheres. Participaram do estudo 10 mulheres (idade $=25,4 \pm 4,6$ anos; massa corporal = 58,8 $\pm 5,1 \mathrm{~kg}$; estatura $=1,61 \pm 0,04 \mathrm{~m}$; índice de massa corporal $=22,6 \pm 1,3 \mathrm{~kg} / \mathrm{m}^{2}$ ), saudáveis, com experiência em treinamento de força de dois meses a dois anos e com faixa etária entre 20 e 33 anos. As participantes realizaram protocolos no modelo crossover. Foram realizadas 4 coletas em dias alternados (48-72 horas) entre cada sessão. Fez-se a avaliação da antropometria e da força muscular (teste de 1RM) na primeira coleta. Nas outras coletas, utilizando o equipamento peck deck, as participantes foram direcionados em ordem aleatória para as seguintes condições experimentais: (a) uma série de 60 segundos de alongamento estático passivo (AEP) seguido por três séries a 70\% de 1RM; (b) uma série de 60 segundos de aquecimento específico seguido por três séries a 70\% de 1RM (AE); (c) uma série de 30 segundos de alongamento estático e uma série de 30 segundos de aquecimento específico $(A E P+A E)$, seguido por três séries a $70 \%$ de $1 R M$. Sendo assim, foi avaliada a resistência muscular localizada, por meio de três séries a $70 \%$ de 1RM, até a falha concêntrica e a PSE ao final de cada série. Anova two-way de medidas repetidas, os testes não paramétricos de Kruskal-Wallis, Friedman e Wilcoxon foram utilizados e o nível de significância foi estabelecido em $p \leq 0,05$. Observou-se que não houve interações significativas entre os protocolos e séries para o desempenho do número de repetições ( $p>0,05)$. Na PSE, não ocorreram diferenças significativas entre os protocolos nas três séries da PSE ( $p>0,05)$ e no somatório da PSE das três séries $(p=0,311)$. Conclui-se que a prática do alongamento estático e/ou aquecimento específico, antecedendo uma sessão de treino de peitoral, parece não influenciar diretamente na quantidade de repetições máximas e PSE em mulheres.
\end{abstract}

PALAVRAS-CHAVE: Exercícios de Alongamento Muscular. Exercício de Aquecimento. Desempenho Físico Funcional. Força Muscular.

Abstract. The aim of the present study was to analyze the acute effect of static stretching and specific warm-up on performance in the number of repetitions and perceived exertion (PE) in women. The participants of the study were ten healthy women ( $58.8 \pm 5.1$ $\mathrm{kg} ; 1.61 \pm 0.04 \mathrm{~m} ; 22.6 \pm 1.3 \mathrm{~kg} / \mathrm{m}^{2}$ ) with strength training background from two months to two years and age ranging from 20 to 33 years old. Participants performed protocols in the crossover model. There were 4 visits on alternate days (48-72 hours) between each session. On the first visit, anthropometry and muscle strength (1RM test) were assessed. On the other visits, using the peck deck equipment, individuals were directed, at random, to the following experimental conditions: (a) a 60-second series of passive static stretching followed by three series at 70\% of 1RM (PSS); (b) a 60-second series of specific warm-up followed by three series at $70 \%$ 1RM (SW); (c) a series of 30 seconds of static stretching and a series of 30 seconds of specific warm-up followed by three series at $70 \%$ of 1RM (PSS+SW). Thus, the localized muscular resistance was assessed through three series at $70 \%$ of 1 RM until the concentric failure and the PE at the end of each series. It was observed that there were no significant interactions between protocols and series for the performance of the number of repetitions $(p>0.05)$. In PE, there were no significant differences between the protocols in the three series $(p>0.05)$ and in the sum of PE in the three series $(p=0.311)$. It is concluded that the practice of static stretching and/or specific warm-up prior to chest training session does not seem to directly influence the number of maximum repetitions and PE in women.

KEYWORDS: Muscle Stretching Exercises. Warm-Up Exercise. Physical Functional Performance. Muscle Strength. 


\section{INTRODUÇÃO}

A aptidão física relacionada à saúde tem como componentes principais a composição corporal, capacidade cardiorrespiratória, flexibilidade, força e resistência muscular localizada. ${ }^{1}$ Estar bem fisicamente não depende apenas de altos níveis de força máxima, mas também de flexibilidade, potência muscular e estabilidade postural, para que assim as atividades diárias sejam realizadas com êxito. ${ }^{2}$

Por isso, é de fundamental importância avaliar estas variáveis, em especial a flexibilidade, antes de intervenções com exercício físico. No entanto, a influência do alongamento muscular e do aquecimento, antes ou depois do exercício, vem trazendo controvérsias no âmbito científico em relação aos seus benefícios, ou prejuízos, no que diz respeito ao desempenho muscular do participante. ${ }^{3}$

O alongamento estático, que é uma forma de otimizar a flexibilidade, consiste em alongar o músculo a máxima amplitude de forma manual, ou mecânica, sustentando a posição por determinado intervalo de tempo. O alongamento estático é o mais utilizado, por ser considerado um método simples, seguro e trazer menos riscos ao praticante. $4,5,6$ Já o aquecimento é definido como qualquer medida de preparação para uma atividade, conhecido como a prática que prepara o organismo para exigências subsequentes. ${ }^{7}$

Diferentes tipos de aquecimento influenciaram positivamente no desempenho de homens adultos treinados, aumentando a performance no teste de repetições máximas. ${ }^{8}$ Já a prática do alongamento estático, dinâmico, ou sem alongamento, não altera a força máxima em adultos. 90 treinamento resistido foi capaz de promover resultados positivos nos testes de força máxima, independente da realização, ou não, do alongamento antes da prática do exercício. ${ }^{10}$

Os protocolos de aquecimento e alongamento estático passivo, utilizados em estudos prévios, não demonstraram diferenças significativas no desempenho muscular, devendo, portanto, serem realizados estudos com protocolos diferentes, mais intensos e extensos. ${ }^{11,12}$ Entretanto, existem lacunas do conhecimento quanto ao efeito agudo do alongamento estático e do aquecimento específico (este que se caracteriza em preparar o corpo para a atividade posterior, utilizando movimentos parecidos com a modalidade pretendida, mas com uma redução na intensidade do trabalho. São exercícios específicos para uma determinada modalidade) e os dois métodos combinados, antecedendo uma sessão de treino de membros superiores com mulheres recreacionalmente treinadas, tomando como base o teste de repetições máximas e a percepção subjetiva de esforço (PSE).

Como não existe um consenso nas pesquisas científicas sobre o efeito do alongamento e aquecimento específico no desempenho do número de repetições e PSE, este estudo procurou esclarecer e ampliar o conhecimento sobre o efeito do alongamento e aquecimento no desempenho físico e percepção subjetiva de mulheres recreacionalmente treinadas. Este conhecimento pode ser útil para subsidiar os atuais programas de treinamento, por ser direcionado aos profissionais que atuam na área da Preparação Física e Avaliação Física.

A hipótese do presente estudo é que não há diferença significativa entre o alongamento estático e/ou aquecimento específico no desempenho do número de repetições e PSE em mulheres recreacionalmente treinadas. Portanto, o 
objetivo foi analisar o efeito agudo do alongamento estático e aquecimento específico no desempenho do número

\section{MATERIAL E MÉTODOS}

\section{Participantes}

Participaram do estudo 10 mulheres (idade $=25,4 \pm 4,6$ anos; massa corporal $=$ 58,8 $\pm 5,1 \mathrm{~kg}$; estatura $=1,61 \pm 0,04 \mathrm{~m}$; índice de massa corporal $=22,6 \pm 1,3 \mathrm{~kg} / \mathrm{m}^{2}$ ), saudáveis, com experiência em treinamento de força de dois meses a dois anos e com faixa etária entre 20 e 33 anos. A dimensão amostral foi realizada utilizando o software $G^{*}$ Power $3.1^{13}$, seguindo os procedimentos propostos por Beck. ${ }^{14}$ Para análise post hoc, foi adotado um $\alpha=0,05$, coeficiente de correlação de 0,5, a correção Nonsphericity de 1 e tamanho de efeito de 0,45 . Portanto, verificou-se que o $\mathrm{n}$ amostral de 10 mulheres é suficiente para fornecer $80,4 \%$ do poder estatístico.

Foram incluídas as participantes que respeitavam os seguintes critérios: I) ser mulher; II) está na faixa etária de 18 a 40 anos; III) não ser hipertensa; IV) ter no mínimo dois meses de experiência e no máximo dois anos, em treinamento de força; $V$ ) responder negativamente todos os critérios do PAR-Q. Foram excluídos os sujeitos que: I) desistiram do estudo por recomendações médica; II) apresentaram alguma lesão osteomuscular. de repetições e PSE em mulheres recreacionalmente treinadas.

\begin{abstract}
Depois de explicar os riscos e benefícios do estudo, as participantes assinaram um termo de consentimento livre esclarecido preparado de acordo com a Declaração de Helsinki. O estudo foi aprovado pelo Comitê de Ética em Pesquisa das Faculdades Nova Esperança (protocolo ${ }^{\circ}$ 244/20 e CAAE: 34459320.6.0000.5179).
\end{abstract}

\section{Desenho do Estudo}

O presente estudo se caracteriza como experimental, com designação aleatória dos sujeitos aos protocolos no modelo crossover. Foram realizadas quatro coletas em dias alternados (48-72 horas) entre cada sessão. Foram avaliadas a antropometria e a força muscular (teste de 1RM), durante a primeira coleta.

Na segunda coleta, as participantes foram direcionadas em ordem aleatória para as seguintes condições experimentais (Figura 1): (a) uma série de 60 segundos de alongamento estático passivo, seguido por três séries a $70 \%$ de $1 \mathrm{RM}$ até a falha concêntrica (AEP); (b) uma série de 60 segundos de aquecimento específico,

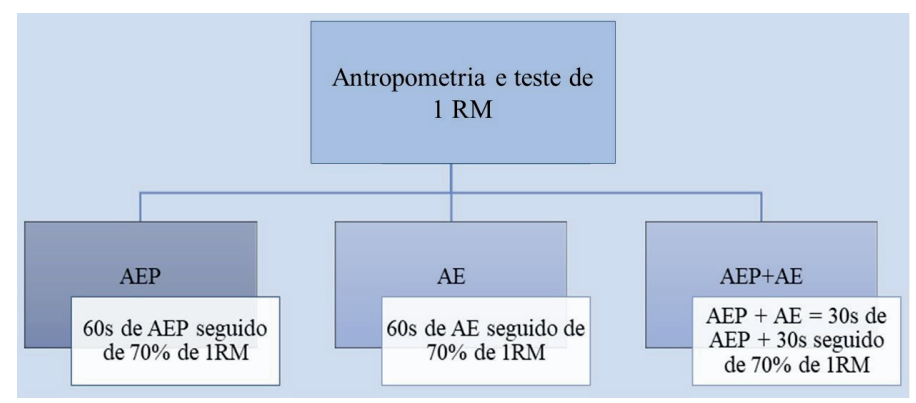

FIGURA 1: Fluxograma do desenho experimental 
seguido por três séries a $70 \%$ de $1 \mathrm{RM}$ até a falha concêntrica (AE); (c) uma série de 30 segundos de alongamento estático passivo e uma série de 30 segundos de aquecimento específico, seguido por três séries a $70 \%$ de $1 R M$ até a falha concêntrica ( $A E P+A E)$.

Durante a coleta de dados foi utilizada uma escala de percepção subjetiva de esforço OMNI-RES15 e um Peck Deck da marca Lion Fitness para realização do exercício. No primeiro encontro, foi realizada de forma referendada a captação das informações da massa corporal $(\mathrm{kg})$ e estatura $(\mathrm{m})$, bem como o teste de $1 \mathrm{RM}$.

\section{Procedimentos}

\section{Teste de Uma Repetição Máxima (1RM)}

Para obtenção da carga máxima, adotou-se o teste de 1RM. O exercício para o músculo peitoral foi realizado de forma bilateral no Peck Deck. Para o aquecimento, cada participante realizou uma série de cinco a dez repetições com 40 e $60 \%$ da força máxima percebida com intervalos de um minuto entre as séries. Depois de um minuto de intervalo, a segunda série foi concluída entre três e cinco repetições a 60-80\% da força máxima percebida. Depois de mais um período de descanso (um minuto), a avaliação da força foi iniciada, na qual poderiam ser feitas até cinco tentativas, ajustando a carga antes de cada nova tentativa.

A duração de recuperação entre as tentativas foi padronizada em 3-5 min. O teste foi interrompido quando a participante não conseguiu executar corretamente $\mathrm{o}$ movimento, sendo considerada a carga máxima a carga mobilizada na última tentativa bem sucedida.

\section{Protocolo de alongamento}

Para $O A E$ foi realizada uma série, mantendo a posição durante 60 segundos em cada série, na qual o movimento acontecia quando um ponto de ligeiro desconforto era atingido. Para o protocolo AEP, fez-se uma série de 60 segundos a $30 \%$ de $1 \mathrm{RM}$, seguido de três séries a $70 \%$ de $1 \mathrm{RM}$ até a falha concêntrica. No protocolo $A E P+A E$, foram realizadas duas séries, sendo uma de 30 segundos de alongamento estático e outra de 30 segundos de aquecimento específico, seguidas de três séries a 70\% de 1RM até a falha concêntrica. Estes procedimentos foram adotados para que o volume de alongamento fosse equalizado entre os protocolos $(A E$, $A E P$ e $A E P+A E$ ).

Em ambos os protocolos de alongamento, praticaram-se exercícios direcionados aos músculos da cintura escapular. O alongamento da cintura escapular acorria da seguinte forma: a voluntária permanecia na posição sentada e realizava uma abdução horizontal da articulação glenoumeral para um ponto de leve desconforto. O movimento foi realizado com os cotovelos flexionados para evitar a insuficiência passiva do músculo bíceps braquial

\section{Protocolo do exercício}

O desempenho da força foi quantificado pelo somatório do número máximo de repetições nas três séries no exercício para o músculo peitoral no Peck Deck (bilateral). O exercício foi realizado com o cotovelo a $90^{\circ}$, com velocidade de execução do movimento de dois segundos (um segundo para ação concêntrica e um segundo para ação excêntrica) até o momento em que ocorresse a falha concêntrica. Quando a participante não conseguia manter o ciclo de repetições dentro da série, na cadência e amplitude estabelecidas, era determinado o ponto de falha concêntrica e o maior número de repetições realizadas com sucesso era computado. 


\section{Avaliação da percepção subjetiva de esforço}

A percepção subjetiva do esforço foi avaliada por meio da escala de OMINIRES15. Consistiu na resposta dada, pelas participantes, à escala, ao final de cada série. Antencedendo a escala, foi feito um processo de ancoragem e memória, para que as participantes a utilizassem de maneira correta.

\section{Análise Estatística}

A normalidade dos dados foi verificada por meio do teste de Shapiro-Wilk e esfericidade de Mauchly's. Anova two-way de medidas repetidas (protocolos $[A L$ vs. $A E$ vs. $A L+A E] \times$ tempo $\left[1^{a}\right.$ série vs. $2^{\text {a }}$ série vs. $3^{\mathrm{a}}$ série $]$ ), seguida pelo teste post hoc de Bonferroni, foi utilizada para a análise de possíveis diferenças no desempenho do número de repetições e para a análise do somatório das séries. Com os testes não paramétricos de Kruskal-Wallis, Friedman e Wilcoxon fez-se a análise da PSE. $O$ nível de significância foi estabelecido em $\mathrm{p} \leq 0,05$. Todas as análises estatísticas foram realizadas utilizando o software estatístico SPSS versão 20.0 do pacote (SPSS Inc., Chicago, IL).

\section{RESULTADOS}

Na análise comparativa pela Two Way ANOVA de medidas repetidas, observou-se que não houve interações significativas entre protocolos $x$ séries $\left(F=0,448 ; n^{2}=0,032 ; p=\right.$ $0,773)$, nos protocolos $\left(F=1,317 ; n^{2}=0,089\right.$; $p=0,285)$ e séries $\left(F=1,356 ; n^{2}=0,048 ; p\right.$
$=0,266) . \mathrm{Na}$ análise comparativa pela Two Way ANOVA, constatou-se que não houve interação significativa estre os protocolos $\left(F=1,317 ; n^{2}=0,089 ; p=0,285\right)$, conforme Tabela 1.

TABELA 1: Análise comparativa das três séries e do somatório total entre os protocolos

\begin{tabular}{ccccc}
\hline \hline \multirow{2}{*}{ Protocolos } & \multicolumn{4}{c}{ Número de Repetições } \\
\cline { 2 - 5 } & $\mathbf{1}^{\text {a Série }}$ & $\mathbf{2}^{\text {a }}$ Série & $\mathbf{3}^{\text {a }}$ Série & Somatório Total \\
\hline $\mathrm{AL}$ & $8,20 \pm 2,20$ & $8,40 \pm 1,71$ & $8,10 \pm 1,79$ & $24,70 \pm 5,31$ \\
$\mathrm{AE}$ & $7,70 \pm 2,66$ & $7,90 \pm 2,23$ & $7,20 \pm 2,29$ & $22,80 \pm 6,94$ \\
$\mathrm{AL}+\mathrm{AE}$ & $9,50 \pm 3,24$ & $9,10 \pm 2,51$ & $9,00 \pm 2,26$ & $27,60 \pm 7,53$ \\
\hline \multicolumn{4}{c}{$\mathrm{AL}=$ Alongamento Estático; $\mathrm{AE}=$ Aquecimento Específico; } \\
& \multicolumn{4}{c}{$\mathrm{AL}+\mathrm{AE}=$ Alongamento Estático + Aquecimento Específico. }
\end{tabular}

Através do teste não paramétrico de Kruskal-Wallis, constatou-se que não há diferenças significativas entre os protocolos nas três séries da PSE $(p>0,05)$ e no somatório da PSE das três séries $(p=0,311)$. No teste de Friedman, observou-se que houve diferença significativa apenas no protocolo que combinou o $A L+A E(p=0,039)$, porém, em uma análise exploratória pelo teste de Wilcoxon, identificou-se que houve uma maior PSE entre a $1^{\mathrm{a}}$ vs. $3^{\mathrm{a}}$ série $(p=0,046)$, conforme Tabela 2.

TABELA 2: Análise comparativa da PSE das três séries e do somatório total da PSE entre os protocolos

\begin{tabular}{|c|c|c|c|c|}
\hline \multirow{2}{*}{ Protocolos } & \multicolumn{4}{|c|}{ Percepção Subjetiva de Esforço (OMNI-RES) } \\
\hline & PSE $1^{a}$ Série & PSE $2^{a}$ Série & PSE $3^{\text {a }}$ Série & PSE Total \\
\hline$\overline{A L}$ & $9,90 \pm 0,31$ & $10,00 \pm 0,00$ & $10,00 \pm 0,00$ & $29,90 \pm 0,31$ \\
\hline $\mathrm{AE}$ & $9,60 \pm 0,69$ & $9,80 \pm 0,42$ & $10,00 \pm 0,00$ & $29,40 \pm 1,07$ \\
\hline $\mathrm{AL}+\mathrm{AE}$ & $9,60 \pm 0,51$ & $9,90 \pm 0,31$ & $10,00 \pm 0,00 *$ & $29,50 \pm 0,70$ \\
\hline
\end{tabular}




\section{DISCUSSÃO}

$O$ presente trabalho analisou o efeito agudo do alongamento estático e aquecimento específico no desempenho no número de repetições e PSE em mulheres recreacionalmente treinadas. Sendo assim, os principais achados foram: 1 ) Os protocolos de alongamento e aquecimento não alteraram significativamente o desempenho do número de repetições; 2) A PSE não foi alterada, após a aplicação dos diferentes protocolos de alongamento, no entanto, a combinação do $A L+A E$ gerou um aumento entre a $1^{a}$ série $v s$. $3^{\mathrm{a}}$ série.

No estudo, observou-se que não houve diferenças significativas no alongamento em relação a resistência muscular localizada. Alguns pesquisadores divergem destes achados. Esses autores constataram que o alongamento, antes do treino de força, pode alterar negativamente essa qualidade física, piorando o rendimento de acordo com o tempo. ${ }^{16,17}$ Tal fato pode ter sido encontrado, devido os estudos terem sido realizados com homens e o presente apenas com mulheres, que, naturalmente, têm um nível maior de flexibilidade, ${ }^{17,18}$ o que pode alterar significativamente os resultados. Sendo assim, a redução no número das repetições máximas, observadas anteriormente ${ }^{16,17}$ podem ter ocorrido em virtude da redução da ativação neural induzida pelo Órgão Tendinoso de Golgi que é situado na junção miotendinosa e responsável por captar elevada força combinada com o alongamento muscular ${ }^{19}$ e, especula-se que isso possa ocorrer com ênfase maior no sexo masculino.

Nesse contexto, o tempo de alongamento realizado no presente estudo pode ter sido baixo para promover alterações na ativação neural das mulheres. Este fato pode justificar a possível similaridade no desempenho do número de repetições entre os métodos de alongamento.

Os estudos de Gallo e Mello ${ }^{8}$ e Tiggeman ${ }^{10}$ trazem resultados que mostram incrementos positivos na força e desempenho muscular, quando uma sessão de treino é antecedida por um aquecimento específico. Confirmam também que, independente da realização ou não do alongamento antes do treino, apenas com a realização do protocolo obteveram-se resultados positivos em relação a força em homens. Tais fatos não foram observados na pesquisa feita com mulheres, o que pode ser dado por algum fator psicológico ou hormonal ${ }^{22}$ que necessitaria ser estudado mais profundamente. Notou-se também que os volumes dos protocolos de alongamento podem não ter gerado estímulo suficiente para gerar fadiga muscular, para que, assim, alterassem o resultado da soma das repetições máximas da coleta. Assim explica-se o número de repetições máximas não terem alterado.

Com relação à PSE, a explicação para o fato de não ter ocorrido diferença entre os protocolos pode estar relacionada ao próprio desenho experimental que realizou as repetições até a falha concêntrica, o que gerou uma elevada PSE para os três protocolos ao final de cada série e no somatório total. No entanto, a combinação do $A L+A E$ gerou um aumento significativo entre a $1^{\text {a }}$ série $v s$. $3^{\text {a }}$ série, assim, uma possível explicação para essa diferença pode ser devido às estruturas viscoelásticas terem sofrido alterações mecânicas por ação do exercício de força que, somada aos efeitos neurais provocados pelo volume do método de alongamento ${ }^{23}$, contribuíram para que o exercício realizado no Peck Deck apresentasse uma maior PSE na $3^{\text {a }}$ série quandocomparado como a $1^{\text {a }}$ série. 
Por fim, o presente estudo tem algumas limitações. A principal limitação deste estudo foi que a arquitetura muscular (ângulo penação, comprimento fascículo ou o tamanho do músculo) não foi examinada, o que poderia explicar ainda mais as

\section{CONCLUSÃO}

A prática do alongamento estático e/ou aquecimento específico, antecedendo uma sessão de treino de peitoral, parece não influenciar diretamente na quantidade de repetições máximas e PSE em mulheres. Notase assim que os profissionais de Educação Física podem aplicar sessões de alongamento

\section{REFERÊNCIAS BIBLIOGRÁFICAS}

1. American College of Sports Medicine. ACSM's health-related physical fitness assessment manual. Lippincott Williams \& Wilkins, 2013.

2. Lafetá JC, Barbosa OS, Jorge JA, Borges L, Coutinho WLM. Correlação entre a aptidão física aeróbica e flexibilidade corpórea de acadêmicos do curso de fisioterapia. Rev. Min. Educ. Fís. 2010; 5(1): 283-293.

3. Ramos GV, Santos RR, Gonçalves A. Influência do alongamento sobre a força muscular: uma breve revisão sobre as possíveis causas. Rev. Bras. Cineantropom. Desempenho Hum. 2007; 9(2): 203-6.

4. Busarelo FO, de Souza FT, de Paula GF, Vieira L, Nakayama GK, Bertolini GRF et al. Ganho de extensibilidade dos músculos isquiotibiais comparando o alongamento estático associado ou não à crioterapia. Fisioter. Mov. 2011; 24(2): 247-254.

5. Pinheiro IM, Góes ALB. Efeitos imediatos do constatações encontradas. Além disso, os resultados escontrtados estão limitados, apenas, para um exercício de grande grupamento muscular dos membros superiores e às mulheres recreacionalmente treinadas. mais volumosos com as mulheres, visto que não se alterou a força muscular e a PSE. Faz-se, portanto, necessária a realização de novos estudos com protocolos de diferentes volumes de alongamento e aquecimento, bem como, com outros grupos musculares. alongamento em diferentes posicionamentos. Fisioter. Mov. 2010; 23(4): 593-603.

6. Morcelli MH, Oliveira JMC, Navega MT. Comparação do alongamento estático, balístico e contrair relaxar nos músculos isquiotibiais. Fisioter. Pesqui. 2013; 20(3): 244-249.

7. Parr M, Price PD, Cleather DJ. Effect of a gluteal activation warm-up on explosive exercise performance. BMJ Open Sport Exerc. Med. 2017; 3(1): 245 .

8. Gallo RC, De Mello WG. Efeitos agudos de diferentes estratégias de aquecimento sobre o desempenho de repetições máximas no exercício de supino reto em homens adultosjovens. Rev. Bras. Presc. Fis. Exerc. 2017; 11(67): 447-452.

9. Oliveira JLS, Gonçalves OS, Nunes MPO, Filho JCCN, Pinto DV, Caminha JSR, Matos, RS, Efeito agudo dos alongamentos estático e dinâmico sobre a produção de força muscular máxima. 
Coleç. Pesqui. Educ. Fís. 2018; 17(4): 63-70.

10. Tiggemann CL, Vanelli C, Molinari T, Kunrath CA, Dias CP. Efeito do alongamento prévio ao treinamento de força no desempenho de força máxima em homens jovens. Biomotriz. 2016; 10(1).

11. Ferreira M, Souza WC, Lima VA, Mascarenhas LPG. Influência do aquecimento no teste de força máxima em mulheres praticantes de musculação. Rev. Inspirar Mov. Saude. 2016; 10(3).

12. Barbosa-Netto S, Almeida MB. Efeito do exercício de alongamento estático passivo contínuo versus fracionado sobre a força muscular. Rev. Andal. Med. Deporte. 2016; 11(1): 1.

13. Faul F, Erdfelder E, Lang AG, Buchner A. G* Power 3: A flexible statistical power analysis program for the social, behavioral, and biomedical sciences. Behav. Res. Methods. 2007; 39(2): 175-191.

14. Beck TW. The importance of a priori sample size estimation in strength and conditioning research. J. Strength Cond. Res. 2013; 27(8): 2323-37.

15. Robertson RJ, Goss FL, Rutkowski J, Lenz B, Dixon C, Timmer J et al. Concurrent validation of the OMNI perceived exertion scale for resistance exercise. Med. Sci. Sports Exerc. 2003; 35(2): 333.

16. Endlich PW, Farina GR, Dambroz C, Gonçalves WLS, Moysés MR, Mill JG et al. Efeitos agudos do alongamento estático no desempenho da força dinâmica em homens jovens. Rev. Bras. Med. Esporte. 2009; 15(3): 200-03.

17. Sá MA, Gomes TM, Bentes CM, Silva GC, Neto $G R$, Novaes JS. Efeito agudo do alongamento estático e facilitação neuromuscular propriocetiva sobre o desempenho do número de repetições máximas em uma sessão de treino de força. Motricidade. 2013; 9(4): 73-81.

18. Bastos CLB, Rosário ACS, Portal MND, Neto GR, Silva AJ, Novaes JS. Influência aguda do alongamento estático no comportamento da força muscular máxima. Motricidade. 2014; 10(2): 90-99.

19. Carvalho ACG, Paula KC, Azevedo TMC, Nóbrega ACL. Relationship between muscular strength and flexibility in healthy adults of both genders. Rev. Bras. Med. Esporte 1998; 4: 2-8.

20. Fowles JR, Sale DG, Macdougall JD. Reduced strength after passive stretch of the human plantarflexors. J. Appl. Physiol. 200; 89(3): 117988.

21. Araújo CGS. Flexibility assessment: normative values for flexitest from 5 to 91 years of age. Arq. Bras. Cardiol.. 2008; 90: 280-87.

22. Jonge XAKJ. Effects of the menstrual cycle on exercise performance. Int. J. Sports Med. 2003; 33(11): 833-51.

23. Lieber R. The physiological basis of rehabilitation: skeletal muscle structure, function, and plasticity. $3^{\mathrm{a}}$. Philadelphia: Lippincott Williams \& Wilkins, 2010. 114-25. 